\title{
Evaluación Tecnológica del Parto de Bajo Riesgo en Diferentes Modelos de Atención
}

\author{
RESULTADOS DEL NIÑO Y COSTOS
}

\author{
'Sonia Pazmiño de Osorio, MD, GO, SP., Carlos Osorio Torres, MD, SO, SP., \\ Melba Franky de Borrero, MD, P, SP., Raúl Bernal Aljure, MD, MI, SP., \\ Vicente Jiménez Velasco, MD, ES, SP.
}

\section{INTRODUCCION}

La presente investigación acerca de la evaluación del niño durante el proceso de atención del parto hasta el mes de nacido, producto de embarazos de bajo riesgo obstétrico, se realizó en tres salas de parto de diferente complejidad en sus recursos físicos y de personal para la atención, en la ciudad de Cali, en 1985 .

Estos diferentes modelos en la atención del parto han sido diseñados para suplir las necesidades de-la población obstétrica pediátrica en la ciudad de Cali y últimamente se han incrementado los esfuerzos a nivel del país, como una estrategia adecuada para cumplir las metas propuestas de "Salud para todos en el año 2000", (1, 2, 3, 4, 5, 6).

Como las evaluaciones de estos modelos han sido básicamente sobre el aspecto administrativo de la utilización del recurso y no se han correlacionado con el impacto en la salud de la madre y el niño, fue el propósito de la investigación medir y comparar los resultados en morbilidad y mortalidad y establecer el costo efectividad de los modelos.

Es el objeto de esta publicación presentar los resultados en el niño y los costos. Los resultados de la madre son objeto de otra publicación (7).

\section{METODOLOGIA}

Se efectuó un estudio prospectivo y analítico que permitió comparar los resultados de morbilidad y mortalidad en el recién nacido producto de gestantes clasificadas como bajo riesgo obstétrico y similar nivel socioeconómico que tuvieron el parto en los tres modelos de atención, que cuentan con diferencias en dotación y en personal, así como en el sistema de apoyo para la solución de las complicaciones que se presentan.

El tiempo de duración del estudio fue de 1 año (agosto de 1984 a julio de 1985).

Se incluyeron las gestantes que consultaron diariamente a cada modelo, durante el mismo período de tiempo, controlando el nivel socioeconómico, la paridad y la edad dentro del rango definido como bajo riesgo obstétrico.

La información la obtuvieron diariamente auxiliares de enfermería con uniformidad de criterio, de la historia clínica, de entrevista con el personal que atendió el parto y de entrevista con la madre.

Al mes de nacido, los niños fueron examinados por médicos según criterios previamente definidos. En caso de duda se practicó examen por pediatra y se practicaron exámenes de laboratorio complementarios.

Durante el período del estudio se calcularon los costos de atención del parto en los tres diferentes modelos.

\section{CARACTERISTICAS DE LOS MODELOS DE ATENCION}

El modelo de menor complejidad (modelo A), pertenece a un centro de Salud del municipio de 
Cali, en donde el parto es atendido por auxiliares de enfermería capacitadas, en una sala con los recursos mínimos necesarios para la atención del parto y del recién nacido. Las complicaciones del recién nacido o el sufrimiento fetal durante el trabajo de parto son remitidas al Hospital Universitario, nivel III de atención.

El modelo de complejidad intermedia (modelo B) pertenece a un centro hospital que se diferencia del anterior en que dispone de una enfermera profesional y un médico general que dan el apoyo inicial a la auxiliar de enfermería cuando se presentan complicaciones durante la atención del parto y período neonatal inmediato y si es necesario remiten el recién nacido al hospital Universitario, nivel III de atención.

El modelo de mayor complejidad (modelo C) pertenece al sistema de la Seguridad Social en donde la responsabilidad del proceso de atención del parto está asignada a médicos especialistas en gineco-obstetricia y la atención del recién nacido a pediatras y neonatólogos que disponen de ayudas diagnósticas y tratamiento de un nivel III de atención.

\section{CARACTERISTICAS DE LAS GESTANTES DE BAJO RIESGO OBSTETRICO}

Se definió como gestantes de bajo riesgo obstétrico las que cumplían las siguientes características:

Edad: Entre 18 y 34 años.

Paridad: Entre 0 y 5 hijos.

Edad de gestación: 37 a 42 semanas.

En los antecedentes obstétricos, que no hubieran presentado:

- Muertes perinatales de menores de ocho días de nacido o mortinato.

- Tres abortos espontáneos sucesivos.

- Cesárea previa.

- Cirugía vaginal o cervical.

_ Intervalo intergenésico menor de seis meses.

- Hemorragia durante el embarazo.
Al examen de ingreso a la sala de partos:

- Que no presentara patología general severa detectable.

- $\quad$ Temperatura menor de $37.6^{\circ} \mathrm{C}$.

_ Tensión diastólica menor de 90 mm de Hg.

- Altura uterina menor de $34 \mathrm{~cm}$.

- Feto único, vivo, fetocardia entre 120 y 160 latidos/minuto.

- Situación longitudinal.

- Presentación cefálica.

- Membranas íntegras o ruptura menor de ocho horas.

Para el análisis de la morbilidad del recién nacido se han clasificado las complicaciones de cada período del parto en dos grupos: severas y otras complicaciones.

Se agruparon como complicaciones severas durante el trabajo de parto: el sufrimiento fetal definido como fetocardia menor de 120 o mayor de 160 latidos por minuto.

Complicaciones severas al parto: recién nacido muerto o con índice de Apgar menor de 7.

Complicaciones severas al post-parto: niño complicado con cualquiera de las siguientes patologías: cianosis, tiraje intercostal, ictericia, hospitalizado.

Complicaciones severas al mes: examen neurológico anormal.

Se agruparon como otras complicaciones, las infecciones e ictericia durante el primer mes de vida.

\section{RESULTADOS EN EL NIÑO}

La gran mayoría de los niños (92.6\%) nacieron normales y no se complicaron durante el proceso del parto, hasta el mes de nacidos, período de observación del estudio.

Del total de complicados $(7,4 \%)$ observamos que los nacidos en el modelo más complejo (C) se 
complicaron el doble $(12.3 \%)$ de los nacidos en el modelo menos complejo (A) (6.2\%) y el triple de los nacidos en el modelo intermedio (B) (3.7\%). Cuadro 1.

\section{CUADRO 1}

PROPORCION DE NINOS COMPLICADOS DURANTE ALGUN PERIODO DEL PARTO

\begin{tabular}{|c|c|c|c|c|}
\hline Niños & Modelo A & Modelo B & Modelo C & Total \\
\hline $\begin{array}{l}\text { Complicados } \\
\text { No complicados }\end{array}$ & $\begin{array}{r}6,2 \\
93,8\end{array}$ & $\begin{array}{r}3,7 \\
96,3\end{array}$ & $\begin{array}{l}12,3 \\
87,7\end{array}$ & $\begin{aligned} 7,4 & (\mathrm{n}=166) \\
92,6 & (\mathrm{n}=2086)\end{aligned}$ \\
\hline TOTAL & $\begin{array}{c}100,0 \\
\mathrm{n}=730\end{array}$ & $\begin{array}{r}100,0 \\
\mathrm{n}=775\end{array}$ & $\begin{array}{r}100,0 \\
n=775\end{array}$ & $\begin{array}{l}100,0 \\
\mathrm{n}=2252\end{array}$ \\
\hline
\end{tabular}

$\begin{array}{lrl}\begin{array}{c}\text { Chi cuadrado con corrección de Yates } \\ \text { (Modelo A }- \text { Modelo B) }\end{array} & 4,21 & \text { G.L.: } 2 \\ \text { Chi }- \text { cuadrado con corrección de Yates }= & 15,87 & 0,01 \\ \quad \text { (Modelo A }- \text { Modelo } \text { ) } & \text { G.L.: } 1\end{array}$

Estas complicaciones más frecuentes en el modelo más complejo $(\mathrm{C})$, se presentaron en el período neonatal en el parto espontáneo, Cuadro 2. Siendo la principal patología la dificultad respiratoria y la cianosis. En el modelo más complejo (C), donde se encontró una mayor intervención en el parto (7) la frecuencia de niños complicados en éstos, es similar a la de los otros dos modelos, sin embargo, los ef ectos a largo plazo deberían estudiarse $(8,9)$.

De los niños complicados en el período neonatal, en el modelo menos complejo (A) el $85.7 \%$ y en el intermedio el $84.6 \%$ no se hospitalizaron, proporciones significativamente mayores que en el modelo más complejo (C) (21.2\%), modelo que permi- te una mayor vigilancia por la disponibilidad del recurso pediátrico, Cuadro 3; y donde se hospitalizan para observación aun niños normales, nacidos de partos intervenidos, siendo el tiempo de hospitalización más frecuente entre 1 y dos días. Dado que el examen al mes del recién nacido no logró detectar trastornos específicos del desarrollo neurológico del niño, sería necesario un seguimiento de la cohorte para determinar los beneficios o no de dicha supervisión.

A pesar de disponer de monitores fetales en el modelo más complejo (C) no se hizo mayor detección de sufrimiento fetal, Cuadro 4, que en los otros dos modelos y la proporción de niños deprimidos con Apgar $\leqslant 6$ fue similar en los tres modelos, Cuadro 5. Luego la mayor detección de trastornos en la evolución del trabajo de parto encontrado en el modelo más complejo (C) (7), no mejoró el resultado en el niño.

La mortalidad perinatal, 1.7 por mil nacidos vivos, como era de esperarse es muy baja en relación con la población general (10). Sin embargo, ocurrieron tres muertes de niños en gestantes del modelo menos complejo (A), una en gestantes del modelo más complejo y 0 en gestantes del modelo intermedio (B).

De los niños muertos de gestantes del modelo menos complejo (A), uno ocurrió por sufrimiento fetal detectado y remitido al hospital de referencia con tardanza en la intervención en esta institución, luego la falla ocurrió en el apoyo brindado al modelo; y dos muertes por circulares del cordón apretados al cuello del niño, que no fueron detectados $\mathrm{y}$ al nacer el niño deprimido fue la auxiliar sola, sin

CUADRO 2

PROPORCION DE NIÑOS CON COMPLICACIONES NEONATALES SEGUN FORMA DE NACIMIENTO

\begin{tabular}{|c|c|c|c|c|c|c|c|c|c|c|}
\hline \multirow{2}{*}{ Forma de nacimiento } & \multicolumn{6}{|c|}{ Niños complicados } & \multicolumn{2}{|c|}{ Total } & \multicolumn{2}{|c|}{$\begin{array}{l}\text { Chi-cuadrado } \\
\text { G.L. }=2\end{array}$} \\
\hline & $\%$ & No. & $\%$ & No. & $\%$ & No. & $\%$ & No. & & \\
\hline Espontáneo & 2,6 & 726 & 1,7 & 770 & 8,1 & 690 & 4,0 & 2186 & 44,49 & $\mathrm{p}<0,01$ \\
\hline Intervenido & 13,3 & 15 & 0 & 8 & 14,5 & 62 & 12,9 & 85 & 1,32 & $\mathrm{p}>0,05$ \\
\hline TOTAL & 2,8 & 741 & 1,7 & 778 & 8,6 & 752 & 4,3 & 2271 & 50,73 & $\mathrm{p}<0,1$ \\
\hline
\end{tabular}


recursos adecuados, quien debió atenderlo y luego trasladarlo sin medios de transporte apropiado al hospital de referencia.

\section{CUADRO 3}

\section{HOSPITALIZACION PARA NINOO COMPLICADO EN EL PERIODO NEONATAL}

\begin{tabular}{|ccccc|}
\hline & $\begin{array}{c}\text { Modelo } \\
\text { Hospitalizado }\end{array}$ & $\begin{array}{c}\text { Modelo } \\
\text { A }\end{array}$ & $\begin{array}{c}\text { Modelo } \\
\text { C }\end{array}$ & Total \\
\hline $\begin{array}{c}\text { No } \\
\text { Si }\end{array}$ & 18 & 11 & 14 & 43 \\
3 & 2 & 52 & 57 \\
\hline TOTAL & 21 & 13 & 66 & 100 \\
\hline $\begin{array}{l}\text { Porcentaje no } \\
\text { hospitalizado }\end{array}$ & 85,7 & 84,6 & 21,2 & 43,0 \\
\hline
\end{tabular}

Chi cuadrado $=37,6 \quad$ G.L. $=2 \quad \mathrm{p}<0,01$

CUADRO 4

PROPORCION DE DIAGNOSTICO

DE SUFRIMIENTO FETAL DURANTE EL TRABAJO DE PARTO

\begin{tabular}{|c|c|c|c|c|}
\hline $\begin{array}{l}\text { Sufrimiento } \\
\text { fetal }\end{array}$ & Modelo A & Modelo B & Modelo C & Total \\
\hline No & 97,5 & 98,5 & 96,2 & $97,4(\mathrm{n}=2215)$ \\
\hline $\mathrm{Si}$ & 2,5 & 1,5 & 3,8 & $2,6(\mathrm{n}=59)$ \\
\hline TOTAL & $\begin{array}{l}100,0 \\
n=727\end{array}$ & $\begin{array}{l}100,0 \\
\mathrm{n}=786\end{array}$ & $\begin{array}{l}100,0 \\
n=761\end{array}$ & $\begin{array}{l}100,0 \\
n=2274\end{array}$ \\
\hline
\end{tabular}

Chi cuadrado $=68,56$

G. L. $=2$

$p>0,01$

CUADRO 5

DISTRIBUCION DEL INDICE DE APGAR A LOS 5 MINUTOS

\begin{tabular}{|cccccl|}
\hline Apgar & Modelo A & Modelo B & Modelo C & Total & \\
\hline $0-6$ & 0,7 & 0,3 & 0,4 & 0,4 & $(\mathrm{n}=10)$ \\
$7-10$ & 99,3 & 99,7 & 99,6 & 99,6 & $(\mathrm{n}=2291)$ \\
\hline \multirow{2}{*}{ TOTAL } & 100,0 & 100,0 & 100,0 & 100,0 \\
& $\mathrm{n}=738$ & $\mathrm{n}=793$ & $\mathrm{n}=770$ & $\mathrm{n}=2301$ \\
\hline
\end{tabular}

Chi cuadrado $=1,65$

G.L. $=2 \quad p>0,01$
La disponibilidad existente de equipos y de especialistas en el modelo más complejo (C), que justifica su mayor costo, Cuadro 6, garantiza una menor mortalidad neonatal; sin embargo, llama la atención la ausencia de muertes en el modelo intermedio, lo que indica cómo la detección oportuna de la complicación y el manejo inmediato brindado, ya sea por la enfermera, parcialmente en el día, o por el médico de forma permanente, pueden definitivamente mejorar los resultados.

\section{CUADRO 6}

COSTOS

\begin{tabular}{|lrrr|}
\hline & Modelo A & Modelo B & \multicolumn{1}{c|}{ Modelo C } \\
\hline & $\$$ & \multicolumn{1}{c|}{$\$$} & \multicolumn{1}{c|}{$\$$} \\
Costo directo & 274.177 & 386.882 & 9.284 .365 \\
Costo indirecto & 152.000 & 52.174 & 1.673 .772 \\
Costo total & 426.177 & 439.056 & 10.958 .137 \\
Costo del parto & 5.395 & 4.181 & 12.983 \\
Costo parto (US) & & & \\
(cambio US 1=102) & 53 & 41 & 127 \\
\hline
\end{tabular}

\section{OTRAS COMPLICACIONES DURANTE EL PRIMER MES}

E1 $9.7 \%$ de los niños presentaron ictericia correspondiente a incompatibilidad de grupo sanguíneo en la gran mayoría de los casos. No hubo diferencias entre los modelos como era de esperarse. No hubo diferencias en las infecciones de la piel, pero la conjuntivitis fue menor en el modelo intermedio (B).

La incidencia de niños de bajo peso fue de $3.8 \%$ menor que en la población de Cali (11); en el modelo más complejo fue aún menor. La proporción de niños con peso mayor de 4.000 gramos fue similar en los modelos y no hubo mayor número de deprimidos en este grupo (12).

\section{Los costos}

El análisis de costos, Cuadro 6, realizado de acuerdo con los registros utilizados en las salas estudiadas demuestra, como era lo esperado, que el modelo más complejo $(\mathrm{C})$, es tres veces más costoso que el modelo intermedio (B) y 2.4 veces que el modelo A. El modelo intermedio (B) demuestra cómo el mejorar el apoyo brindado con personal más capacitado que el modelo A, no significa aumentar el costo por parto, puesto que éste depende más del número de partos atendidos y sí mejora los resultados de morbilidad en la madre y el niño. 


\section{CONCLUSIONES Y RECOMENDACIONES}

De la evaluación realizada en tres diferentes modelos de complejidad para la atención del parto de bajo riesgo obstétrico, considerando únicamente los resultados en el niño y los costos de atención del parto, que es el objeto de esta publicación, se encontró que la disponibilidad de monitores fetales en el modelo más complejo (C) no mejoró la detección del sufrimiento fetal durante el trabajo de parto ni mejoró los resultados en la frecuencia de la presión al nacer (resultados de Apgar).

La mayor frecuencia de complicaciones se encontró en el período neonatal inmediato, la dificultad respiratoria y la cianosis, en el modelo más complejo (C), sin embargo; como la evaluación al mes de nacimiento no detectó diferencias clínicas entre los niños de los tres modelos, sería necesario tener un tiempo mayor para repetir estas observaciones.

En el modelo de complejidad intermedia (B) en donde se dispone de personal de mayor capacidad técnica (médico general y enfermera) para la interconsulta inmediata, que en el modelo menos complejo (A), no se presentó mortalidad neonatal, obteniéndose por lo tanto los mejores resultados en este modelo.

El costo de la atención del parto del modelo (B) también resultó ser el más económico, dada su utilización, y dados los resultados en el niño, el más efectivo.

\section{RESUMEN}

Se presentan los resultados en el niño, hasta el primer mes de nacidos, y los costos, de la evaluación tecnológica de tres modelos de Atención del Parto, con diferente nivel de complejidad, en 2.313 gestantes de bajo riesgo obstétrico y similar nivel socioeconómico, en Cali, Colombia.

Son estos resultados, en conjunto con los de la madre, objeto de otra publicación, los que determinan la escogencia del mejor modelo.

Se hace énfasis en que las conclusiones comprenden a modelos de atención en una población urbana y a muy corta distancia (5-10 minutos) del hospital de referencia.

\section{CONCLUSIONES Y RECOMENDACIONES GENERALES DEL ESTUDIO}

Con fines de la publicación, fue necesario presentar los resultados del estudio en dos artículos; los resultados maternos y los resultados del niño y costos. Sin embargo como para la Evaluación de los modelos de atención los tres tipos de resultados son indispensables para las conclusiones finales, hemos creído conveniente que cada artículo lleve las conclusiones y recomendaciones del estudio.

De la evaluación realizada en tres diferentes modelos de complejidad para la atención del parto de bajo riesgo obstétrico, se ha encontrado que el modelo intermedio (B) de un centro hospital que dispone, además del personal auxiliar de enfermería, de una enfermera y de médico institucional que comparte sus otras responsabilidades de atención médica permanente con las llamadas de interconsulta de la sala de maternidad, es el menos costoso, dada la atención de mayor número de partos al año, y a la vez el más efectivo en el período de observación del estudio que incluyó hasta 1 mes después del parto.

Presentó sin embargo, mayor sangrado de las gestantes que debe analizarse y corregir sus causas. A pesar de que el modelo menos complejo (A) presentó resultados maternos similares al modelo B se requiere mejorar tanto el apoyo de equipo y personal en la sala como el sistema de referencia para disminuir la mortalidad neonatal.

En el modelo más complejo $(\mathrm{C})$, se evidenció una mayor detección de patología materna y neonatal que es necesario estudiar en posteriores trabajos para aclarar sus causas y las posibles implicaciones en el desarrollo neurológico del niño a largo plazo incluyendo los tres modelos.

Debe tenerse presente que estas conclusiones son para modelos de atención de una población urbana y a muy corta distancia (5-10 minutos) del hospital de referencia.

Para el diseño de modelos simplificados de atención del parto debe tenerse presente que a estas salas consultan en trabajo de parto gestantes de alto riesgo $(26.2 \%)$ cuyos resultados en la morbilidad y mortalidad no se midieron en este estudio y que las gestantes de bajo riesgo obstétrico o su hijo presentaron complicaciones durante el proceso de atención del parto en un $30 \%$. El tipo de complicaciones propias del trabajo de parto y parto hacen 
necesario que los diferentes modelos de atención afinen su sistema de intervención para el manejo adecuado de la complicación de la madre o el niño.
También se debe recomendar el diseño de un sistema permanente de evaluación y seguimiento de las madres y sus hijos usuarios de estas salas.

\section{AGRADECIMIENTOS}

Esta investigación fue financiada por la Oficina Panamericana de la Salud, a quien presentamos nuestros sentimientos de gratitud y muy especialmente a los doctores Gloria Coe, - Arturo Romero, David Banta, Jorge Peña, Juan César García (q. e. p. d.) y Ricardo Schwarz y el grupo del Centro Latinoamericano de Perinatología (CLAP).
Agradecemos además la colaboración de todo el personal, tanto administrativo como operativo, que labora en las tres salas incluidas en el estudio, y al señor Reynaldo Carvajal, nuestro estadístico por su valiosa colaboración.

\section{BIBLIOGRAFIA}

1. OFICINA SANITARIA PANAMERICANA. Conferencia Taller Inter-regional sobre tecnología apropiada para la Salud. Bol. OPS, V. 92, No. 6. pp. 529-531, junio de 1982.

2. SUAREZ OJEDA, E.N. El enfoque de riesgo en la atención perinatal y materno infantil. Bol. OPS, Vo. 92, No. 6, p. 482, junio 1982.

3. ORGANizACiON MUNDIAL DE LA SALUd. Método de atención sanitaria de la madre y el niño basado en el concepto de riesgo. Publicación en Offset No. 39, Ginebra, 1978.

4. DELGADO, H. Modelo integrado y simplificado de servicios de salud, nutrición y planificación familiar para zonas rurales. Bol. OPS, V. 94, No. 4, pág. 362-378. abril, 1983.

5. - Extensión de la cobertura de los servicios de salud gracias a las parteras tradicionales: Un decenio de progreso. Crónica de la OMS, V. 36, No. 3, pp. 100-105, 1982.

6. WALLACE, C.; MARShaL, F.; CHARTRUSET, R. Examen retrospectivo de los servicios médicos materno-infantiles en el hospital Albert Schweitzer en el Haití rural. Bol. OPS, V. 92, No. 5, p. 391, 1982.

7. PAZMIÑO DE OSORIO, S.; OSORIO, C.; FRANKY DE BORRERO, M.; BERNAL, R.; JIMENEZ, V. Evaluación tecno- lógica del parto de bajo riesgo en diferentes modelos de atención. Resultados maternos. Bol. OPS.

8. SCANLON, J.W. Efectos de analgesia y anestesia en el neonato. Clínicas obstétricas y ginecológicas. V. 2, p. 649, 1981.

9. INGARDIA, J.C.; CERTULOS, C.L.Uso y abuso del fórceps. Clínicas de perinatología. Parto y expulsión difíciles. V. 1, p. 63, 1981.

10. PAZMIÑo DE OSORIO, S., PEREZ, F.; OSORIO, C.; BARRETO, J.; GOMEZ, J. y demás colaboradores. Atención materno infantil. Rev. Col. de Obst. y Ginec. V. 33, No. 6 p. 376, Nov. - Dic. 1982.

11. REY, V.H. El bajo peso en la salud pública. El recién nacido latinoamericano, 1a. Edición. Talleres gráficos de Impresora Feriva Ltda., p. 163, Cali.

12. HOUCHANG, D.; MODAN, L.; WNEDY, L.; DORCHESTER, THOROSIAN, A.; FREEMAN, R.K. Macrosomía; maternal, fetal and neonatal implications. Obst. \& Gynecol. V. 55, No. 4, Ap. 1980, p. 420-424, April 1980. 